\title{
XXX.
}

\section{Zur Casuistik der Lymphcysten des Oberschenkels.}

\author{
Von \\ Dr. Achilles Nordmann in Basel.
}

Die folgende Mittheilung will über eine seltene Beobachtung von Oberschenkelgeschwulst berichten, der völlig Gleichartiges, meiner Litteraturkennntniss nach, nicht an die Seite zu stellen sein dürfte.

Frau W., 34 Jahre alt, ist von jeglicher hereditärer Belastung frei und, Influenza ausgenommen, früher nie schwer krank gewesen. Sie hat drei Mal geboren. Erste Geburt 8. December 1892, zweite 15. August 1894, dritte 24. April 1897. Von bei der ersten Geburt zurtickgebliebenen Placentarresten, die eine Uterusausräumung nöthig machten, und einer Dammnaht abgesehen, völlig normaler Verlauf der Entbindungen sowohl wie der Wochenbette. Die drei Kinder leben und sind gesund.

Ihre gegenwärtigen Beschwerden führt die Patientin auf einen Fall zuruck, den sie Ende November 1892, wenige Tage vor ilırer ersten Niederkunft, erlitten hatte, bei dem sie ausgeglitscht und ziemlich heftig auf die rechte Gesässhälfte gefallen war. Sie legte dem Vorkommniss anfänglich keine Bedeutung bei, wurde aber durch Sclimerzen, die sie beim Aufstehen nach der Geburt in der Gesässgegend empfand, unliebsam daran erinnert. Während der folgenden Jahre verschwanden diese Schmerzen nie vollständig, sie wurden ärztlicherseits auf tiefliegende Krampfadern zurückgefuhrt und mit Einreibungen behandelt.

Vor 6 Jahren, also etwa 1 Jahr nach dem Trauma, bemerkte die Frau zum ersten Mal an der ursprünglich betroffenen Stelle eine Geschwust, die Anfangs gänseei-, später handtellergross wurde, beim Wachsthum sich nach unten senkte und ohne irgend welche andere Empfindungen zu bewirken, beim Gehen immer mehr hinderlich wurde.

Im Februar 1899, zur Zeit der Aufnahme dieser Beobachtung, deckt die Geschwulst die ganze hintere Fläche des rechten Oberschenkels und reicht vom Trochanter major angefangen bis in die Kniekehle herab. Die Haut über derselben ist leicht verschieblich, nicht ödematös und von spärlichen ectatischen Venen durchzogen. Die Geschwulst erscheint auf der Unterlage leicht beweglich, weist eine prall elastische Consistenz und stellenweise dunkle Fluctuation auf. Thre Oberfäche ist völlig glatt, nirgends besonders differenzirt. Entzlindliche Erscheinungen irgend welcher Art und Oedem der Umgebung fehlen vollständig. Die Musculatur des 
Oberschenkels functionirt normal und weist mit der Geschwulst keinen Zusammenhang auf.

Die Diagnose schwankte $z$ wischen Lipom und Cyste und neigte wegen der Seltenheit der letzteren Geschwulstform an dieser Stelle mehr der ersteren Möglichkeit zu.

Operation am 10. Februar 1899 unter freundlicher Assistenz des Herrn Dr. Markees. Ohne Benutzung der Esmarch'schen Blutleere wird in Chloroformnarkose durch einen vom Trochanter bis zur Kniekehle reichenden, über die Vorwölbung der Geschwulst hinwegziehenden Schnitt nach Durchtrennung des subcutanen Fettes die bläulich durchscheinende Geschwulst freigelegt, die sich als enorme Cyste darstellt. Sie wird mit Leichtigkeit aus dem subcutanen Fett herausgeschält. Nach der Tiefe, beziehungsweise nach vorn hin reicht sie bis auf die Oberschenkelmusculatur, von der sie sich ebenso leicht trennen lässt. Nach oben findet sie ihr Ende genau unterhalb des grossen Trochanter, wo sie wie durch ein etwa $3 \mathrm{~cm}$ langes fasciales Band am Trochanter befestigt ist, beziehungsweise durch dieses Band mil der Oberschenkelfascie zusammenhängt. Die Auslösung der Geschwulst ging so leicht von statten, dass während und nach derselben, uberhaupt bei der ganzen Operation eine Unterbindung nicht nöthig wurde. Nach Entfernung derselben lag die Musculatur der hinteren Oberschenkelfläche ohne irgend welche fasciale Bedeckung bloss zu Tage. Fortsätze der Geschwulst oder ihrer Wandung zwischen die einzelnen Muskeln sind nicht vorhanden. Naht der etwa $27 \mathrm{~cm}$ langen Hautwunde. Drainage durch den unteren Wundwinkel.

Nicht so einfach wie die Operation gestaltete sich der spätere Verlauf. Beim Verbandwechsel am 7. Tage erschien zwar die Wundlinie durch eine tadellose Prima geheilt und auch die Oeffnung für den Drain, der am 4. Tage entfernt worden war, verklebt, der Heilungsvorgang beim Mangel jeglicher Temperatursteigerung somit zunächst ideal. Drei Tage später aber fand sich bei intacter äusserer Wundlinie die Haut in weitem Umkreis sackartig aufgebläht und fluctuirend. Bei Druck auf dieselbe entleerte sich aus dem unteren Wundwinkel eine reichliche, nicht gemessene, etwas blutig tingirte, dünne Flitssigkeit, nach deren Entleerung die Hautauftreibung verschwunden war. Zudem bestanden leichte Fieberregungen. Der gleiche Vorgang wiederholte sich bei jedem Verbandwechsel während der folgenden Woche. Kein Zweifel, dass es sich hier um einen echten Lymphfluss handelte, dessen Beseitigung anzustreben war.

Da die Patientin einen weiteren blutigen Eingriff verweigerte, konnte von einer Wiederöffnung der Wunde zunächst keine Rede sein. Bei dem völlig reizlosen Zustand der Höhle wurde deshalb versucht, eine entzündliche Reizung herbeizufuhren and dadurch den Verschluss der Lymphgefässe einzuleiten. Ausspülungen mit Jod- und Höllensteinlösungen blieben erfolglos, und ebensowenig nutzten Tamponaden mit verschiedenen Gazearten. Erst durch die Injection einer 10 proc. Zinkchloridlösung gelang es des Lymphflusses Herr zu werden. Dabei entstanden durch eine auch sonst bekannte Wirkung dieser Substanz kleinere nekrotische Herde im Fettgewebe und der Muskelsubstanz, die unter Temperatursteigerung zu umschriebener Abscessbildung und zu einer Perforation der Hautnarbe 
Veranlassung gaben. Eine jedenfalls auf die gleiche Ursache zurückzufubrende, tiefer liegende Eiteransammlung in der Musculatur gab späterhin zu einer grösseren Spaltung der Hautnarbe Veranlassung. Nach und nach und nachdem oberflächliche Fistelgänge, die das Anlegen der Haut verhinderten, gespalten worden waren, verheilte die Wunde per granulationem, ohne dass der Lymphfluss wiedergekehrt wäre, und ohne dass die Musculatur des Oberschenkels irgend welchen Schaden gelitten hätte.

Untersuchung der Geschwulst: Dieselbe stellt eine einkamme. rige, in den grössten Durchmessern $21 \mathrm{~cm}$ lange und $11 \mathrm{~cm}$ breite Cyste dar, bei deren Beschreibung zwischen Cystenwand und Cysteninhalt zu unterscheiden ist.

Die Cystenwand ist auf der Aussenfläche an vielen Stellen von der Oberschenkelmusculatur angehörigen Muskelfasern und von lockerem Bindegewebe eingehulltt. Die Innenfläche stellt eine im Ganzen glatte Fläche dar, auf der sich nur wenige, kurze, brückenartige Stränge und kleine bis hirsekorngrosse Excrescenzen vorfinden. Die Wand ist etwa $21 / 2 \mathrm{~mm}$ dick und zeigt schon makroskopisch eine gewisse Schichtung.

Bei der mikroskopischen Untersuchung tritt dieselbe noch deutlicher hervor. Man erkennt dabei mehrere Lagen bald locker gefitgter, bald dichter aneinander liegender Bindegewebsfibrillen, zwischen welche namentlich gegen die Cysteninnenfläche zu zahlreiche, zellige Elemente einge. lagert sind; zumeist sind es Spindelzellen, vielfach aber findet man auch Rundzellen, die stellenweise ganze Nester bilden. Die Cysteninnenfläche ist an den meisten Stellen von einer mehrschichtigen Lage endothelähnlicher Zellen ausgekleidet, die nur an wenigen Punkten fehlt, an welchen die Cysteninnenfläcle von Bindegewebe begrenzt wird. - Zwischen den Bindegewebsbündeln, d. h. in dem lockeren Zwischengewebe finden sich Anhäufungen von Fettträubchen. - Die papillären Excrescenzen zeigen den gleichen histologischen Bau wie die übrige Cystenwand. Epithel ist nirgends vorhanden.

Der Inhalt der Cyste, der leider nicht vollständig aufgefangen werden konnte, beläuft sich auf etwa $1 \frac{1}{2}$ Liter. Es ist eine citronengelbe, klare, alkalische Flussigkeit, in welcher schon makroskopisch erkennbare Cholestearinkrystalle herumschwimmen. Er reagirt alkalisch und gerinnt beim Kochen. Beim längeren Stehen bilden sich im Bodensatz spärliche, weiche Gerinnsel. Mikroskopisch finden sich in ilım ausser den schon erwähnten Cholestearintafeln Rundzellen, Fetttröpfehen, Körnchen- und Spindelzellen.

Der gegebenen Schilderung nach handelt es sich in diesem Fall um eine mit endothelähnlichen Zellen ausgekleidete Cyste, für deren Deutung am einfachsten von ihrem Inhalt ausgegangen wird. Derselbe ist, auch ohne dass eine quantitative Analyse stattgefunden hätte, und ohne dass aus der während der Heilung abgehenden Flüssigkeit Rückschlüsse gezogen würden, genügend als Lymphe charakterisirt.

Die Lymphe findet sich in einem Hohlraum, der an die Stelle der normalen Oberschenkelfascie, der Fascia lata getreten ist. Es geht 
dies mit Sicherheit daraus hervor, dass nach Wegnahme der Cyste die blosse Oberschenkelmusculatur frei zu Tage tritt, und dass die Cyste an ihrem oberen Ende in der Gegend des Trochanter major in das normaler Weise vorhandene Fascienblatt übergeht.

Die Entstehung der Cyste wird wohl auf eine natürliche Weise durch den von der Patientin seiner Zeit erlittenen Fall erklärt. In Folge desselben kam es zu einer Zerreissung der Fascie unterhalb des Trochanter und jedenfalls auch zur Zerreissung grösserer Lymphgefässe, aus welchen alsdann continuirlich Lymphe in das fasciale Gewebe hineinsickerte. Im Laufe der Zeit, allerdings auffallend langsam, wurden die Schichten desselben immer mehr auseinandergedrängt, es bildete sich nach und nach ein grosser Sack, dessen Wandungen secundär hypertrophirten und die beschriebene Form annahmen. Die Rundzelleninfiltration sowie der Spindelzellenreichthum der Cystenwand machen es wahrscheinlich, dass man es hier mit einem in Proliferation befindlichen Gewebe zu thun hat.

Die Operation des Falles betreffend ist vielleicht darin gefehlt worden, dass die Cyste da, wo sie in das Fascienblatt über dem Trochanter überging, einfach herausgeschält und das zurückbleibende Fascienband nicht unterbunden wurde. Es ist möglich, dass bei diesem Verfahren die den Heilungsverlauf so sehr complicirende Lymphorrhagie ausgeblieben wäre.

Die Geschwulst dürfte auf Grund dieser Darstellung als ein Kystoma lymphaticum fasciale vielleicht nicht unrichtig bezeichnet sein.

Aehnliche Beobachtungen sind in der Litteratur nur spärlich vorzufinden, ganz identische feblen gänzlich.

König 1) spricht von seltenen Fällen, in welchen Cysten des Oberschenkels zu grossen bis in die Nähe des Knies reichenden Geschwülsten sich zu entwickeln vermögen. Die Casuistik Michel's ${ }^{2}$ ), auf die er sich hierbei bezieht, betrifft Geschwülste der vorderen und inneren Fläche des Oberschenkels, die bis ins kleine Becken hinein sich verfolgen liessen und mit der geschilderten Beobachtung keine Analogien aufweisen. Die herangezogenen von Padien ${ }^{3}$ ) veröffentlichten Fälle V elpeau's gehören, wie aus dem Studium des Originals mit Sicherheit hervorgeht, überhaupt nicht hierher.

1) König, Lehrbuch der Chirurgie. 7. Auflage. 1900. IUI. S. 561.

2) Michel, American journal of medical science. 1879. January.

3) Padieu, Bourses séreuses et leurs maladies. Thèse de Paris. 1839. 
576 XXX. Nondmakn, Zur Casuistik der Lympheysten des Oberschenkels.

Bei den neueren Beobachtungen Narath's 1) und Strehl's 2) handelt es sich in ähnlicher Weise wie bei Michel um herabgetretene retroperitoneale Lymphcysten oder um tuberculöse Senkungsabscesse.

Am nächsten steht der beschriebene Fall den Befunden von Lymphextravasaten, über die Morel-Lavallée ${ }^{3}$ ) zuerst genaue Untersuchungen veröffentlicht und über die Guss en bau e ${ }^{4}$ ) in neuerer Zeit zusammenhängend berichtet hat. Fünf der von diesen Autoren beschriebenen dreizehn Fälle betreffen ebenfalls den Oberschenkel und sind nach ihnen so entstanden, dass in Folge eines tangentialen, durch ein Trauma ausgeübten Druckes eine Zerreissung zwischen Haut und oberflächlicher Fascie stattfand, die eine Lymphextravasation zur Folge hatte.

In meinem Fall muss die Zerreissung, wie aus der Beschreibung hervorgeht, innerbalb der Fascie selbst stattgefunden und zur Theilung des Fascienblattes geführt haben. Dieser Umstand erklärt vielleicht das überaus langsame Wachsthum der Geschwulst im Gegensatze zu den rasch zunehmenden zwisehen Haut und Fascie gelegenen Lymphextravasaten, deren Ausdehnung kein so starres Hinderniss wie das aponeurotische Bindegewe entgegenwirkte. Als besondere Eigen. thümlichkeit bleibt bei meinem Fall hervorzuheben, dass hier das Lymphextravasat secundär zur Bildung einer völlig geschlossenen Cyste geführt hat, ein Vorkommniss, das ich sonst nirgends mitgetheilt finde und das, wenn der Vorgang richtig gedeutet ist, ein seltenes Beispiel traumatischer Cystenbildung darstellen würde.

1) Narath, Archiv f. klin. Chirurgie. Bd. L. S. $763 \mathrm{ff}$.

2) Strehl, Zeitschr. f. Chirurgie. Bd. I.I. S. $178 \mathrm{ff}$.

3) Morel-Lavallée, Archives générales de médecine. 1853. Vième série, tome I, p. $691 \mathrm{ff}$. und ibid. 1863. VIième série, tome $\mathrm{I}$, p. $20 \mathrm{ff}$.

4) Gussenbauer, Deutsehe Chirurgie. Liefermg 15. S. $114 \mathrm{ff}$. 